\title{
Wesentliche Änderungen zu ISO 50001:2011
}

Die ISO 50001:2018 entstand nach den Revisionen im Bereich Qualitätsund Umweltmanagement. Wesentliche Änderungen zur ursprünglichen ISO 50001:2011 sind:

- Übernahme der High-Level-Structure

- Aktualisierung der Definitionen

- Ausweitung der Betrachtung geltender und eingegangener Verpflichtungen

- Stärkung der Verantwortlichkeit der Leitungsebene

- Wegfall der Forderung nach einem Managementbeauftragten

- Stärkung der Forderung nach einem Energieteam

- Pflicht zur Veröffentlichung der Energiepolitik bei Bedarf

- Forderung der Planung von Maßnahmen zum Umgang mit Chancen und Risiken

- Stärkerer Fokus auf die Analyse der wesentlichen Bereiche

- Ausweitung des Datenerfassungskonzepts

- Einführung des Konzeptes der dokumentierten Information

- Integration des Managementsystems in die Geschäftsprozesse

- Forderung nach einem Auditprogramm

- Der kontinuierliche Verbesserungsprozess (KVP) wird zur fortlaufenden Verbesserung 